\title{
65 kpc of ionized gas trailing behind NGC 4848 during its first crossing of the Coma cluster ${ }^{\star}$
}

\author{
Matteo Fossati ${ }^{1}$, Giuseppe Gavazzi ${ }^{1}$, Alessandro Boselli ${ }^{2}$, and Michele Fumagalli ${ }^{3}$ \\ 1 Università degli Studi di Milano-Bicocca, Piazza della Scienza 3, 20126 Milano, Italy \\ e-mail: [matteo.fossati; giuseppe.gavazzi]@mib.infn.it \\ 2 Laboratoire d'Astrophysique de Marseille, UMR 6110 CNRS, 38 rue F. Joliot-Curie, 13388 Marseille, France \\ e-mail: alessandro.boselli@oamp.fr \\ 3 Department of Astronomy and Astrophysics, University of California, 1156 High Street, Santa Cruz, CA 95064, USA \\ e-mail: mfumagalli@ucolick.org
}

Received 2 July 2012 / Accepted 18 July 2012

\begin{abstract}
In a five hour $\mathrm{H} \alpha$ exposure of the northwest region of the Coma cluster with the $2.1 \mathrm{~m}$ telescope at San Pedro Martir (Mx), we discovered a $65 \mathrm{kpc}$ cometary emission of ionized gas trailing behind the SBab galaxy NGC 4848. The tail points in the opposite direction of the cluster center, in the same direction where stripped HI had been detected in previous observations. The galaxy shows bright HII regions in an inner ring-like pattern, where the star formation takes place at the prodigious rate of $\sim 8.9 M_{\odot} \mathrm{yr}^{-1}$. From the morphologies of the galaxy and the trailing material, we infer that the galaxy is suffering from ram pressure due to its high velocity motion through the intergalactic medium. We estimate that $\sim 4 \times 10^{9} M_{\odot}$ of gas is swept out from the galaxy forming the tail. Given the ambient conditions in the Coma cluster $\left(\rho_{0}=6.3 \times 10^{-27} \mathrm{~g} \mathrm{~cm}^{-3} ; \sigma_{\text {vel }}=940 \mathrm{~km} \mathrm{~s}^{-1}\right)$, simulations predict that the ram pressure mechanism is able to remove such an amount of gas in less than 200 Myr. This, combined with the geometry of the interaction, is indicative of radial infall into the cluster, leading to the conclusion that NGC 4848 has been caught during its first passage through the dense cluster environment.
\end{abstract}

Key words. galaxies: clusters: individual: Coma - galaxies: individual: NGC 4848 - galaxies: ISM - galaxies: interactions

\section{Introduction}

Yagi et al. (2010) reported a deep (4.5 h integration) $\mathrm{H} \alpha$ survey covering the central $0.5 \times 0.5 \mathrm{deg}^{2}$ of the Coma cluster (A1656) with the Suprime-Cam mounted on the Subaru telescope. Unexpectedly for an evolved cluster such as Coma, these authors found that almost every star-forming member has its own spectacular complex of diffuse, ionized, gaseous trails extending dozens of kpc behind the optical extent of the galaxies, and sometimes harboring star-forming compact knots. They revealed 14 such systems, including those previously reported by Yagi et al. (2007) and Yoshida et al. (2008) in the Coma cluster. Similar examples can also be found in A1367 (Gavazzi et al. 2001), in Virgo (Yoshida et al. 2002, 2004; Kenney et al. 2008), and in A3627 (Sun et al. 2007). These features suggest that the galaxies were recently captured by the cluster gravitational potential and are now infalling toward the cluster center (Yagi et al. 2010). Unfortunately, the field of view of the Suprime-Cam missed by less than 5 arcmin the position of NGC 4848, which is another obvious candidate for possible extended $\mathrm{H} \alpha$ emission. Bothum \& Dressler (1986) had indeed listed NGC 4848 as one of the dozen unusually active galaxies found in the Coma cluster.

NGC 4848 (CGCG 160-055; Zwicky et al. 1961-1968) is a bright $\left(M_{B}=-20.5\right)$ SBab:edge-on (RC3, de Vaucouleurs et al. 1991) galaxy that lies at the northwest (N-W) periphery of the $\mathrm{X}$-ray emitting region in the Coma cluster. It has a vigorous

\footnotetext{
* Observations taken at the observatory of San Pedro Martir (Baja California, Mexico), belonging to the Mexican Observatorio Astronómico Nacional.
}

star-formation rate (SFR) of $\sim 9 M_{\odot} \mathrm{yr}^{-1}$ as derived from the $\mathrm{H} \alpha$, ultraviolet (UV), far-infrared (FIR), and radio-continuum emission (see Sect. 3). Observations in the $21 \mathrm{~cm}$ line of HI (Gavazzi 1989; Bravo Alfaro et al. 2001) revealed a moderately deficient $\mathrm{HI}$ content $\left(\operatorname{Def}_{\mathrm{HI}}=0.46\right)$, displaced in the N-W direction, as opposed to its $\mathrm{H}_{2}$ content, which appears normal and centrally concentrated (Vollmer et al. 2001). The asymmetry in the HI distribution suggests that the galaxy is experiencing ram pressure (Gunn \& Gott 1972) owing to its high velocity motion through the intergalactic medium (IGM). This discrepancy is expected since unless ram pressure stripping is severe, only the atomic phase of the gas distributed at the galaxy periphery is removed, while the $\mathrm{H}_{2}$, bound deep within the galaxy potential well, is mostly unaffected by ram pressure (Combes et al. 1988; Kenney \& Young 1989; Boselli et al. 2002; Fumagalli \& Gavazzi 2008).

Numerical hydrodynamical simulations of galaxies subject to ram pressure stripping in rich clusters (e.g. Kapferer et al. 2009; Tonnesen \& Bryan 2009, 2010, 2012; Ruszkowski et al. 2012) reveal that in much less than 1 Gyr these galaxies lose all of their gas when the density of the IGM and the transit velocity are as high as in the Coma cluster. Consistent results are found both with and without magnetic fields. Extended gaseous tails form and the gas is shocked and heated by turbulence (Yoshida et al. 2004, 2012; Kenney et al. 2008), producing compact knots where radiative cooling takes place favoring the star formation.

In 2000, we serendipitously discovered a low surfacebrightness $\mathrm{H} \alpha$ emission trailing behind NGC 4848 (not reported by Iglesias-Páramo et al. 2002) in a one-hour exposure of the central $1 \times 1 \mathrm{deg}^{2}$ of the Coma cluster with the Wide Field 
Table 1. Log book of the imaging observations.

\begin{tabular}{lccccccc}
\hline \hline Telescope & Date & CCD & $\begin{array}{c}\text { Pix } \\
(\operatorname{arcsec})\end{array}$ & $\begin{array}{c}\text { Filter } \\
(\AA)\end{array}$ & $\begin{array}{c}\text { Tint } \\
(\mathrm{s})\end{array}$ & Nexp & $\begin{array}{c}\text { Seeing } \\
(\operatorname{arcsec})\end{array}$ \\
\hline INT & 20 Mar. 1999 & $4 \times 2048 \times 4100 \mathrm{EEV}$ & 0.33 & $B$ & 300 & 2 & 1.0 \\
INT & 28 Apr. 2000 & $4 \times 2048 \times 4100 \mathrm{EEV}$ & 0.33 & $6725(80)$ & 1200 & 3 & 1.3 \\
INT & 28 Apr. 2000 & $4 \times 2048 \times 4100 \mathrm{EEV}$ & 0.33 & $(\mathrm{Gunn}) r^{\prime}$ & 300 & 3 & 1.3 \\
TNG & 09 Feb. 2001 & $1024 \times 1024 \mathrm{NICS}$ & 0.25 & $H$ & 60 & 9 & 0.8 \\
SPM & Apr. 2012 & $1024 \times 1024 \mathrm{EEV}$ & 0.35 & $6723(80)$ & 600 & 30 & 1.4 \\
SPM & Apr. 2012 & $1024 \times 1024 \mathrm{EEV}$ & 0.35 & $($ Gunn $) r$ & 60 & 26 & 1.4 \\
\hline
\end{tabular}

Camera (WFC) at the Isaac Newton Telescope (INT, La Palma). However, this extended emission was only marginally detected and follow-up observations were required. Similar extended features were detected in deep GALEX images by Smith et al. (2010) showing several knots of recent star-formation along the tail. In 2012, we acquired additional five-hour observations with the San Pedro Martir (SPM) telescope using narrow-band $\mathrm{H} \alpha$ filters. The resulting stacked six-hour exposure, which we present in this work, is sufficiently deep to allow a robust determination of the flux in the tail. Throughout this paper, we assume $H_{0}=73 \mathrm{~km} \mathrm{~s}^{-1} \mathrm{Mpc}^{-1}$, thus NGC 4848 is at the distance of $95.5 \mathrm{Mpc}$, that of the Coma cluster.

\section{Observations}

We observed NGC 4848 in the $B$ band using the WFC at the prime focus of the $2.5 \mathrm{~m}$ INT in 1999 and in the $H$ band using the Near Infrared Camera Spectrometer (NICS) mounted at the $3.6 \mathrm{~m}$ Telescopio Nazionale Galileo (TNG) in 2001. Both observations (available through GoldMine, Gavazzi et al. 2003) were obtained under $\leq 1.0$ arcsec seeing (see Table 1). We also observed the field centered on NGC 4848 in $\mathrm{H} \alpha$ using two telescopes: the INT, and the $2.1 \mathrm{~m}$ telescope at SPM. The observations were performed through narrow-band filters centered at $\sim 6725 \AA$, covering the redshifted $\mathrm{H} \alpha$ and [NII] lines (ONband image). The underlying continuum was measured through the broad-band $r$-Gunn filter (OFF-band image; see Table 1 for details). The images were obtained in photometric conditions with seeing ranging from 1.3 to $1.4 \mathrm{arcsec}$. To minimize the impact of cosmic rays, we acquired a set of shorter exposures that were subsequently combined using a median filter. Images of the spectrophotometric star Feige 34 were also collected for the photometric calibration.

The individual images were bias-subtracted and flat-fielded using combinations of exposures of several empty fields at twilight. A second-order flat-field correction was achieved using superflat images obtained by median-combining several science exposures that were selected because these are the ones containing only point-like objects among all exposures taken during the 2012 run. The images were aligned using field stars as reference (using the IRAF task imalign) and fitted individually with third-order polynomials to correct for residual flat-field inhomogeneities (using the IRAF task imsurfit). After background subtraction and rebinning the two sets of images to a common pixel scale of 0.35 arcsec pixel $^{-1}$ (using the IRAF task imlintran), we obtained the final combined image (using the IRAF task imcombine). The resulting stacked frames correspond in total to a $6 \mathrm{~h}$ and $41 \mathrm{~min}$ integration for the ON-band and OFF-band, respectively.

Finally, we derive the continuum-subtracted $\mathrm{H} \alpha+[\mathrm{NII}]$ image in the following way. First, the intensity in the combined
Table 2. Physical parameters of NGC 4848 that complement Table 1 of Vollmer et al. (2001).

\begin{tabular}{lc}
\hline \hline$\alpha(\mathrm{J} 2000)$ & $12^{\mathrm{h}} 58^{\mathrm{m}} 05^{\mathrm{s}} .6$ \\
$\delta(\mathrm{J} 2000)$ & $28^{\circ} 14^{\prime} 34^{\prime \prime}$ \\
Distance $^{a}$ & $95.5 \mathrm{Mpc}$ \\
$u^{b}$ & $15.82 \mathrm{mag}$ \\
$g^{b}$ & $14.54 \mathrm{mag}$ \\
$r^{b}$ & $13.81 \mathrm{mag}$ \\
$i^{b}$ & $13.42 \mathrm{mag}$ \\
$z^{b}$ & $13.17 \mathrm{mag}$ \\
$M_{\text {Dyn }}{ }^{b}$ & $3.8 \times 10^{11} M_{\odot}$ \\
$M_{\text {Star }}{ }^{b}$ & $4.8 \times 10^{10} M_{\odot}$ \\
$M_{\mathrm{HI}}{ }^{b}$ & $2.2 \times 10^{9} M_{\odot}$ \\
$M_{\mathrm{H}_{2}}{ }^{f}$ & $4.1 \times 10^{9} M_{\odot}$ \\
$M_{\text {trail }}{ }^{g}$ & $3.6 \times 10^{9} M_{\odot}$ \\
$M_{\text {Bar }}{ }^{h}$ & $5.8 \times 10^{10} M_{\odot}$ \\
$M_{\text {Dyn }} / M_{\text {Bar }}$ & 6.5 \\
$S F R^{g}$ & $8.9 M_{\odot} \mathrm{yr}^{-1}$ \\
\hline \multicolumn{2}{c}{} \\
\hline
\end{tabular}

Notes. ${ }^{(a)}$ Assuming a distance modulus $m-M=34.9$ mag (Gavazzi et al. 2010). ${ }^{(b)}$ Petrosian AB magnitudes from the SDSS DR7 database (Abazajian et al. 2009). Corrected for extinction in the Galaxy (Schlegel et al. 1998). ${ }^{(c)}$ Assuming centrifugal equilibrium, $V_{\text {rot }}=270 \mathrm{~km} \mathrm{~s}^{-1}$ (Amram et al. 1992) and the $B$-band diameter $a_{25}=1.6 \operatorname{arcmin}(\mathrm{RC} 3$, de Vaucouleurs et al. 1991). ${ }^{(d)}$ Derived from the $i$ magnitudes and $g-i$ color using a modified Bell et al. (2003) recipe consistent with the mass determination of MPA-JHU. ${ }^{(e)}$ Gavazzi (1989). ${ }^{(f)}$ Vollmer et al. (2001). ${ }^{(g)}$ This work. ${ }^{(h)} M_{\mathrm{Bar}}=M_{\mathrm{Star}}+M_{\mathrm{HI}}+M_{\mathrm{H}_{2}}+M_{\text {tail }}$.

OFF-band image was normalized to that of the combined ON-band one, using the flux ratio of several field stars. Then, since Spector et al. (2012) pointed out that the colors of foreground stars differing from those in the target galaxies can be an important source of error when estimating the normalization coefficient, we applied a correction as follows. We evaluated that the average color of NGC 4848 is $g-r_{\text {gal }}=0.7$ mag (see Table 2). This is only slightly different from the mean color of Milky Way stars at similar Galactic latitude $g-r_{\text {stars }}=0.8$ mag (Spector et al. 2012). From the linear fit shown in Fig. 2 of Spector et al. (2012), we infer that the normalization coefficient for NGC 4848 is 0.98 times the one estimated from the foreground stars. In our case, this correction is only marginal. The combined NET-image was then obtained by subtracting the normalized OFF-band frame from the ON-band one. The resulting OFF-band and NET frames are shown in Fig. 1. The limiting $\mathrm{H} \alpha$ surface brightness was estimated using photometry of randomly sampled apertures of 8 arcsec in diameter. The resulting $1 \sigma$ limiting sensitivity is $1.0 \times 10^{-17} \mathrm{erg} \mathrm{cm}^{-2} \mathrm{~s}^{-1} \operatorname{arcsec}^{-2}$ (at full resolution), which is a factor of four shallower than the superb Subaru data from Yagi et al. (2010). 

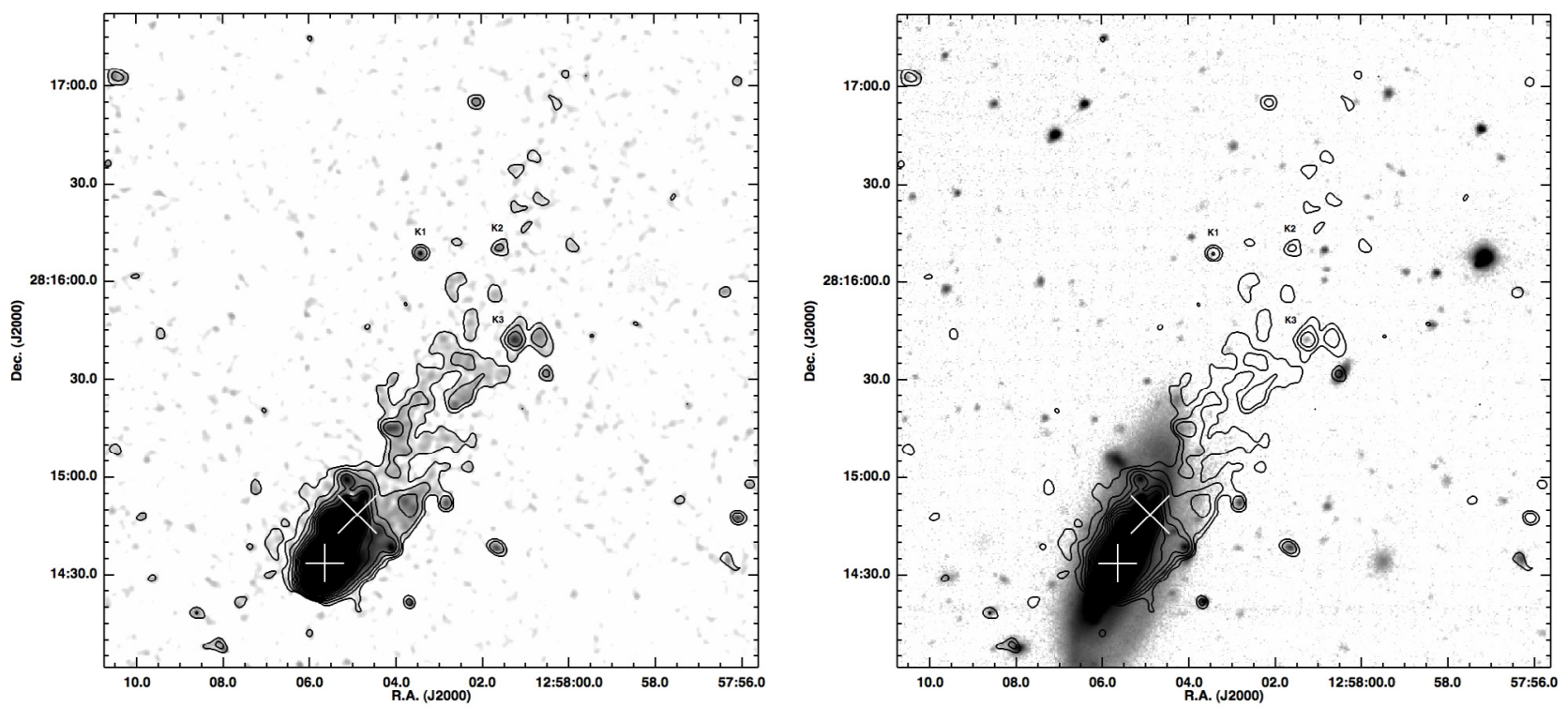

Fig. 1. Left panel: grayscale representation of the Gaussian-smoothed (three-pixel-kernel) NET image of NGC 4848, with contours of the same image (contours are from 1 to $320 \times 10^{-17} \mathrm{erg} \mathrm{cm}^{-2} \mathrm{~s}^{-1} \operatorname{arcsec}^{-2}$ in 10 logarithmic intervals). The white cross marks the center of the molecular hydrogen given by Vollmer et al. (2001), while the white X marks the center of the HI given by Bravo Alfaro et al. (2001). Three knots of NET emission with associated stellar continuum are marked K1-3. Right panel: grayscale representation of the OFF image of NGC 4848, with contours of the Gaussian-smoothed (three-pixel-kernel) NET image (same as left panel). Note the angle between the major axis of the galaxy and the direction marked by the tail, which points toward the center of the Coma cluster.

\section{The galaxy}

In spite of its SBab classification, the morphology of NGC 4848 is rather irregular, being composed of a central nuclear source (harboring an active galactic nucleus (AGN) [NII] $\lambda 6584 \AA / \mathrm{H} \alpha=$ 0.64 , see Gavazzi et al. 2011) with red $(g-i=1.5 \mathrm{mag})$ color. At a 5-10 arcsec projected radial distance from the nucleus, a bright, blue, and clumpy ring of active HII regions dominates the galaxy morphology. Further out, the light profile falls-off exponentially, as is typical of a disk. Given the irregular shape of the galaxy, a simple (bulge+disk) model cannot fit the measured light profile in any band. Therefore, a non-parametric classification method has to be used, such as the CAS system of Conselice (2003).

Based on the $\mathrm{H} \alpha$ and $r$-band images obtained in 2012 and on $B$ and $H$-band images, we derived the concentration, asymmetry and clumpiness (CAS) parameters of NGC 4848 (see Table 3). We remind the reader that a theoretical $r^{1 / 4}$ law has $C=5.2$, while an exponential disk has $C=2.7$. Moreover, a symmetric and smooth galaxy has $A=0, S=0$. Typical values for $\mathrm{Sa}-\mathrm{Sb}$ galaxies in the $r$ band are $C=3.9 \pm 0.5$, $A=0.07 \pm 0.04$, and $S=0.08 \pm 0.08$ (Conselice 2003), which are consistent with the values of $C=3.0 \pm 0.5, A=0.11 \pm 0.05$, and $S=0.10 \pm 0.10$ found by Fossati et al. (in prep.) for a larger sample of 360 galaxies in the Coma Supercluster in the $r$ band. The same set of parameters were derived in the NET H $\alpha$ frames, yielding $C=2.5 \pm 0.9, A=0.28 \pm 0.11$, and $S=0.65 \pm 0.26$ for $\mathrm{Sa}-\mathrm{Sb}$. Therefore, NGC 4848 is significantly more asymmetric and clumpy and less concentrated than typical Sab galaxies, especially in bands that more sensitively trace the presence of young stars, consistently suggesting that some morphological peculiarity has been recently $(<1 \mathrm{Gyr})$ induced by the interaction of the galaxy with its surrounding environment.

\subsection{The SFR from the $\mathrm{H} \alpha$ luminosity}

To make a quantitative estimate of the $\mathrm{H} \alpha+[\mathrm{NII}]$ flux associated with NGC 4848, we preferred to use the new SPM image alone
Table 3. Concentration, asymmetry, and clumpiness (CAS) parameters of the galaxy NGC 4848 in four bands.

\begin{tabular}{lccc}
\hline \hline Band & $C$ & $A$ & $S$ \\
\hline $\mathrm{H} \alpha$ & 1.76 & 1.05 & 1.75 \\
$\mathrm{~B}$ & 1.83 & 0.45 & 1.06 \\
$r$ & 2.50 & 0.30 & 0.57 \\
$\mathrm{H}$ & 3.06 & 0.18 & 0.35 \\
\hline
\end{tabular}

to avoid uncertainties caused by to resampling that occurs when the image is stacked with the existing INT observation. The $\mathrm{H} \alpha+[\mathrm{NII}]$ flux within the optical extent of NGC 4848 was found to be $\log F(\mathrm{H} \alpha+[\mathrm{NII}])=-12.47 \pm 0.03 \mathrm{erg} \mathrm{cm}^{-2} \mathrm{~s}^{-1}$. Before converting this flux into a SFR, the $\mathrm{H} \alpha$ flux must be corrected for Galactic extinction, contamination due to [NII] $\lambda \lambda 6548,6584 \AA$, internal extinction, and dust extinction (see Boselli et al. 2009; Gavazzi et al. 2012). Given the high Galactic latitude of the Coma cluster, extinction due to the Milky Way (Schlegel et al. $1998)$ is negligible (the dust-corrected value is $\log F(\mathrm{H} \alpha+$

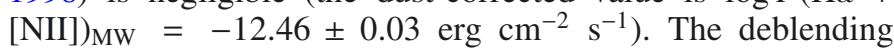
from [NII] was computed using the drift-scan spectrum of the galaxy taken from Gavazzi et al. (2004). The corrected flux became $\log F(\mathrm{H} \alpha)_{\mathrm{MW} ; \mathrm{DB}}=-12.68 \pm 0.03 \mathrm{erg} \mathrm{cm}^{-2} \mathrm{~s}^{-1}$. The flux was further corrected for internal extinction using the Balmer decrement (as in Boselli et al. 2012), which was determined again with the drift-scan spectrum to yield $\log F(\mathrm{H} \alpha)_{\mathrm{MW} ; \mathrm{DB} ; \mathrm{AA}}=$ $-12.11 \pm 0.03 \mathrm{erg} \mathrm{cm}^{-2} \mathrm{~s}^{-1}$. The final correction for dust absorption in HII regions assumes that only a fraction $f$ of the Lyman continuum photons contribute to the ionization of the atomic hydrogen. Following Boselli et al. (2009) and assuming a Salpeter IMF, $f=0.77$ we obtained $\log F(\mathrm{H} \alpha)_{\text {MW;DB;AA;EX }}=$ $-11.99 \pm 0.03 \mathrm{erg} \mathrm{cm}^{-2} \mathrm{~s}^{-1}$. Conversely, the photon escape fraction was considered to be negligible (Boselli et al. 2009). The corrected $\mathrm{H} \alpha$ luminosity was thus $L_{\mathrm{H} \alpha}=1.1 \pm 0.2 \times 10^{42} \mathrm{erg} \mathrm{s}^{-1}$. According to Kennicutt (1998), the unobscured SFR is given by $S F R_{\mathrm{H} \alpha}\left[M_{\odot} \mathrm{yr}^{-1}\right]=7.9 \times 10^{-42} \times L_{\mathrm{H} \alpha}\left[\mathrm{erg} \mathrm{s}^{-1}\right]$, thus $S F R_{\mathrm{H} \alpha}=$ $8.8 \pm 0.2 M_{\odot} \mathrm{yr}^{-1}$. The bulk of the star formation takes place in 
bright HII regions distributed along the central ring-like pattern, as discussed above (see Fig. 2 Left).

\subsection{The SFR from the UV luminosity}

Using Kennicutt (1998) $S F R_{\mathrm{UV}}\left[M_{\odot} \mathrm{yr}^{-1}\right]=1.4 \times 10^{-28} \times$ $L_{\mathrm{UV}}\left[\mathrm{erg} \mathrm{s}^{-1} \mathrm{~Hz}^{-1}\right.$, where $L_{\mathrm{UV}}$ is obtained following Boselli (2011) from the GALEX far-ultraviolet (FUV) magnitude of 16.29 mag (Martin et al. 2005). The UV flux is particularly affected by dust extinction and the correction $(A(F U V)=$ $2.01 \mathrm{mag}$ ) is obtained following Cortese et al. (2008) assuming that there is a balance between the absorbed UV photons and the total FIR $(3-1100 \mu \mathrm{m})$ energy. From the corrected UV magnitude, we obtained $S F R_{\mathrm{UV}}=10.7 M_{\odot} \mathrm{yr}^{-1}$.

\subsection{The SFR from the FIR luminosity}

According to Helou et al. (1985), the FIR 8-1000 $\mu$ m luminosity is obtained by combining the $12,25,60$, and $100 \mu \mathrm{m}$ flux from IRAS (Neugebauer et al. 1984). Since NGC 4848 is undetected in the IRAS $12 \mu \mathrm{m}$ band, we used the WISE (Wright et al. 2010) $12 \mu \mathrm{m}$ flux. We obtained $F_{\mathrm{IR}}=1.51 \times 10^{-13} \mathrm{Wm}^{-2}$. Using the Kennicutt (1998) calibration $S F R_{\mathrm{IR}}\left[M_{\odot} \mathrm{yr}^{-1}\right]=4.5 \times 10^{-44} \times$ $L_{\mathrm{IR}}\left[\mathrm{erg} \mathrm{s}^{-1}\right]$, we obtained $S F R_{\mathrm{IR}}=7.4 M_{\odot} \mathrm{yr}^{-1}$.

\subsection{The SFR from the radio continuum luminosity}

The estimate of the SFR from the radio continuum (RC) is based on the $1415 \mathrm{MHz}$ flux (21.8 mJy) from NVSS (Condon et al. 2002) corrected for the nuclear contribution (7.1 mJy) from FIRST (Becker et al. 1994). Following the Bell et al. (2003) calibration, $S F R_{\mathrm{RC}}\left[M_{\odot} \mathrm{yr}^{-1}\right]=5.52 \times 10^{-22} \times L_{\mathrm{RC}}\left[\mathrm{W} \mathrm{Hz}{ }^{-1}\right]$, we obtained $S F R_{\mathrm{RC}}=8.8 M_{\odot} \mathrm{yr}^{-1}$.

Assuming that the star formation is constant during the last $10^{7}(\mathrm{H} \alpha)-10^{8}(\mathrm{UV}, \mathrm{FIR}, \mathrm{RC}) \mathrm{yr}$ (Iglesias-Páramo et al. 2004), the average SFR becomes $S F R=8.9 \pm 1.1 M_{\odot} \mathrm{yr}^{-1}$, corresponding to a specific SFR (SSFR) of $1.9 \times 10^{-10} \mathrm{yr}^{-1}$. This is more than a factor of ten higher than the SSFR of galaxies of similar mass and Hubble type. In conclusion, the galaxy appears to be undergoing a starburst phase possibly triggered by the interaction with the cluster environment (as claimed by Bekki \& Couch 2003).

\section{4. $\mathrm{H} \alpha$ tail parameters}

The flux estimate of the low-brightness tail requires a careful assessment of the quality of the flat-fielding and background subtraction. For extended sources, the dominant source of error is associated with the background variations on scales similar to those of the source. We measured the residual background in several $20 \times 20 \operatorname{arcsec}^{2}$ regions (comparable with the extension of the trail) within the field and determined that the median of the residual background on this scale corresponds to $10 \%$ of the background rms. From this figure, we estimated the uncertainty in the the $\mathrm{H} \alpha$ flux in the tail (see Gavazzi et al. 2012).

At the distance of the Coma cluster, the total extent of the tail of $2.25 \times 0.66 \mathrm{arcmin}^{2}$ corresponds to $62.5 \times 18.5 \mathrm{kpc}$. To estimate the total flux in the tail, we integrated the counts above the 2- $\sigma$ level in the full-resolution SPM image alone inside a polygonal region of $2.25 \times 0.66 \mathrm{arcmin}^{2}$, excluding the galaxy itself and the compact knots (see below) (the polygonal region is sketched in Fig. 2). We obtained $\log F_{\text {tail }}=-14.40(-14.66) \pm$ $0.12 \mathrm{erg} \mathrm{cm}^{-2} \mathrm{~s}^{-1}$. Hereafter, the value in parenthesis is obtained by correcting for $[\mathrm{NII}] \lambda \lambda 6548,6584 \AA$ contamination when a
$[\mathrm{NII}] / \mathrm{H} \alpha$ ratio of 0.62 is assumed, as for the main galaxy ${ }^{1}$. No correction for internal extinction was applied to the tail.

The line intensity of the low-brightness tail was obtained using

$I_{\mathrm{H} \alpha}=\frac{F_{\text {tail }}}{h v_{\mathrm{H} \alpha} \times \Omega}=0.13(0.07)$ Rayleigh

$\left(1\right.$ Rayleigh $=10^{6} / 4 \pi$ photons $\left.\mathrm{cm}^{-2} \mathrm{~s}^{-1} \mathrm{sr}^{-1}\right)$. Following Spitzer (1978), the $\mathrm{H} \alpha$ line radiation from an optically thin nebula at $T=10^{4} \mathrm{~K}$ is customarily expressed in terms of the emission measure (EM). Adopting an "effective recombination coefficient" of $\alpha_{32}=1.17 \times 10^{-13} \mathrm{~cm}^{3} \mathrm{~s}^{-1}$ (Osterbrock 1989), we derived $1 \mathrm{Ry}_{\mathrm{H} \alpha}=2.77 \mathrm{EM}_{\mathrm{H} \alpha}$.

In the case of NGC 4848, EM amounts to 0.36 (0.19) $\mathrm{cm}^{-6}$ pc.

Assuming that the tail has cylindrical symmetry, this implies a mass of ionized hydrogen of $5.0 \times 10^{9}\left(3.6 \times 10^{9}\right) M_{\odot}$, thus a plasma density of $4.3 \times 10^{-3}\left(3.2 \times 10^{-3}\right) \mathrm{cm}^{-3}$. If the filling factor is $<1$, the values obtained should be taken as an upper limit to the mass and a lower limit to the density. The plasma in the tail must have originally consisted of neutral hydrogen, therefore a rough estimate of the total gas loss from the galaxy can be derived using the HI deficiency parameter defined by Giovanelli \& Haynes (1985). The galaxy contains $2.2 \times 10^{9} M_{\odot}$ (Gavazzi 1989), corresponding to an HI deficiency parameter of 0.46 , implying that $4.1 \times 10^{9} M_{\odot}$ of gas has been left behind in the tail. This estimate is consistent with the mass previously found in the ionized gas.

We assumed a transit velocity through the cluster of $\sim 1330 \mathrm{~km} \mathrm{~s}^{-1}$, as derived from $\sqrt{2} \sigma_{\text {vel }}$ (where we also assumed that the cluster can be modeled as an isothermal sphere, as in Cayatte et al. 1994), where $\sigma_{\text {vel }}=940 \mathrm{~km} \mathrm{~s}^{-1}$ for A1656 (Gavazzi et al. 2010). From the length of the tail, we then derived that the ionized material survived some $10^{7.6} \mathrm{yr}$. The recombination time $\tau_{\mathrm{r}}=1 / N_{\mathrm{e}} \alpha_{32}$, is $10^{7.8}\left(10^{7.9}\right) \mathrm{yr}$, i.e. consistent with the survival time. Although recent simulations favor a scenario where the gas is heated in situ to $\mathrm{H} \alpha$ emitting temperatures and the eddies along the tail contain sufficient turbulent energy to currently sustain its ionization (Tonnesen \& Bryan 2010), it cannot be excluded that part of the gas in the tail was ionized by the galaxy HII regions or AGN.

In addition to the ionized diffuse gas, the tail of NGC 4848 harbors some compact star-forming regions (possibly HII regions) that we label K1-3 in Fig. 1. They are visible on the $r$ band image and they coincide with compact features in the FUV image by Smith et al. (2010). Their uncorrected $\mathrm{H} \alpha+[\mathrm{NII}]$ flux is $3.6 \times 10^{-16}, 2.1 \times 10^{-16}$, and $7.4 \times 10^{-16} \mathrm{erg} \mathrm{cm}^{-2} \mathrm{~s}^{-1}$ respectively. This corresponds to a luminosity of $4 \times 10^{38}, 2.3 \times 10^{38}$, and $8 \times 10^{38} \mathrm{erg} \mathrm{s}^{-1}$, which are typical of HII regions, and represent $\sim 25 \%$ of the total luminosity in the tail. Similar "fireballs" are found in the wakes of other stripped galaxies, such as GMP3016 and GMP4060 in the Coma cluster (Yagi et al. 2010), or either VCC 1217 (Fumagalli et al. 2011) and VCC 1249 (Arrigoni Battaia et al. 2012) in the Virgo cluster. These authors claim that these HII regions were recently born in situ out of stripped material, as predicted by some hydrodynamical simulations (e.g. Kapferer et al. 2009; Tonnesen \& Bryan 2012).

Boissier et al. (in prep.) claim the absence of in situ star formation behind galaxies in the Virgo cluster displaying HI

\footnotetext{
1 The presence of a central AGN might lead to an overestimate of the $[\mathrm{NII}] / \mathrm{H} \alpha$ ratio. However, Yoshida et al. (2012) found that this ratio in the filaments associated with IC4040, another giant spiral galaxy in the Coma cluster, is that of a LINER-like spectrum.
} 

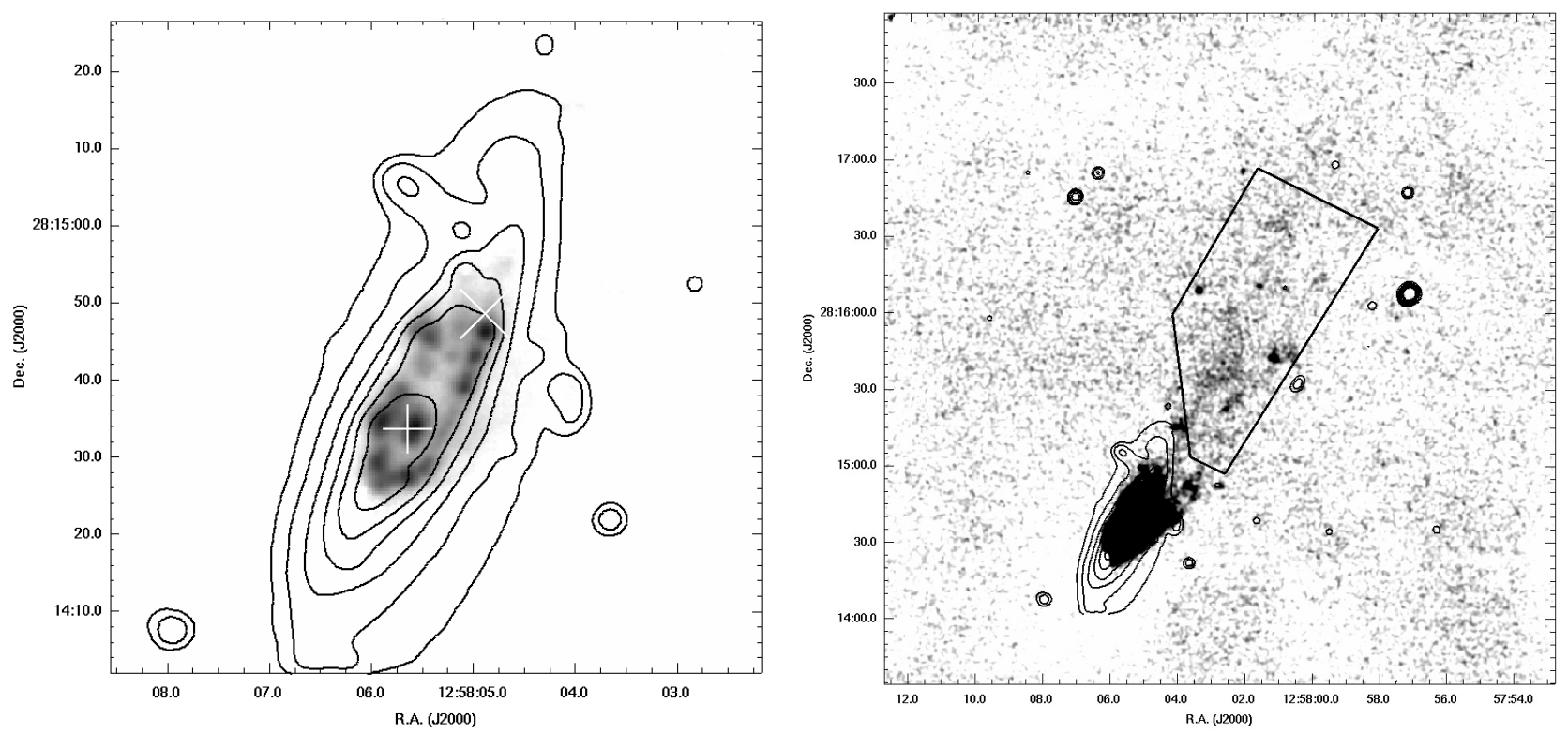

Fig. 2. Left panel: grayscale representation of the inner structure of the NET image of NGC 4848 at full resolution, with contours of the OFF-band image. The white cross marks the center of the molecular hydrogen given by Vollmer et al. (2001), while the white X marks the center of the HI given by Bravo Alfaro et al. (2001). Right panel: high-contrast representation of the Gaussian-smoothed (three-pixel-kernel) NET image to highlight the emission in the tail, with contours of the OFF-band image. The black box marks the region where the flux in the tail was computed.

(Chung et al. 2007) but no $\mathrm{H} \alpha$ tails. As explicitly mentioned by Tonnesen \& Bryan (2010, 2012), the pressure by the IGM (e.g. density, velocity dispersion) is crucial for determining the formation of relevant eddies that might increase the IGM density enough to provide the cooling conditions driving the formation of molecular clouds, thus of stars. Such conditions are met in the Coma cluster and near the center of Virgo, but not further out where most of the Boissier et al. objects are located.

\section{Discussion}

There is unanimous consensus (Yagi et al. 2010; Yoshida et al. 2012) that the morphological disturbances suffered by several late-type galaxies in the Coma cluster are caused by the dynamical interaction with the IGM, namely by the ram pressure mechanism (Gunn \& Gott 1972), including NGC 4848 (Gavazzi 1989; Vollmer et al. 2001). The question is how long this process has been acting for and whether the galaxy is infalling into the cluster for the first time or, as maintained by Vollmer et al. (2001), it entered the cluster environment about 1 Gyr ago and already crossed the cluster center $400 \mathrm{Myr}$ ago.

From the length of the tail, we inferred that the galaxy motion takes place primarily in the plane of the sky, probably at $V \sim 1330 \mathrm{~km} \mathrm{~s}^{-1}\left(\sqrt{2} \sigma_{\text {vel }}\right)$. Unfortunately, we cannot directly measure this velocity since from the redshift we can infer only the component along the line of sight $\left(V_{/ /}\right)$. Subtracting from the heliocentric velocity of NGC 4848 (Zabludoff et al. 1993) the mean velocity of Coma cluster galaxies (Gavazzi et al. 2010), we obtained $V_{/ /}=257 \mathrm{~km} \mathrm{~s}^{-1}$.

There are two possibilities: that the orbit is circular, with its angular momentum in the plane of the sky, or that it is radial. In the first case, the ratio of the velocity along the line of sight to the tangential velocity $V_{/ /} / V=257 / 1330=0.19$ gives the angle between $V_{\perp}$ and $V$. Assuming that the present projected radial distance of NGC 4848 from the cluster center is $0.75 \mathrm{Mpc}$, we conclude that the radius of the circular orbit is $4 \mathrm{Mpc}$, i.e. exceeds the virial radius of Coma by almost a factor of two. We are much more likely to conclude, however, that the orbit is radial or at least highly eccentric, and that the present motion of NGC 4848 is toward the center of the cluster at $V \sim 1330 \mathrm{~km} \mathrm{~s}^{-1}$, as suggested by the direction of the $\mathrm{H} \alpha$ tail pointing in the opposite direction.

More evidence of infall into the Coma cluster is provided by the velocity distribution of late-type galaxies (LTG), which in general hardly follow a Gaussian distribution but one that is skewed toward either lower or higher velocities than the overall mean velocity (Biviano et al. 1997; Boselli \& Gavazzi 2006). The velocity dispersion profile of the LTGs in clusters is found to be consistent with orbits more radial than those of early-type galaxies (ETG), providing a picture in which possibly all spirals have not yet crossed the virialized cluster core, and may even be on a first (infall) approach toward the central, high-density region.

In the ram pressure scenario, the amount of stripped gas can be computed from the equilibrium between the gravitational binding energy and the dynamical pressure. The calculation requires the density profile of the gas in the IGM. Following Cavaliere \& Fusco-Femiano (1978), the density radial profile is well-approximated by an isothermal sphere

$\rho_{\mathrm{IGM}}=\rho_{0}\left[1+\left(\frac{r}{r_{\mathrm{c}}}\right)^{2}\right]^{-3 \beta / 2}$,

where $\rho_{0}$ represents the central density and $r_{\mathrm{c}}$ the scale-length. Using the parameters estimates of Mohr et al. (1999) for the Coma cluster (all quantities having been recomputed for $H_{0}=$ $73 \mathrm{~km} \mathrm{~s}^{-1} \mathrm{Mpc}^{-1}$ ) namely $\rho_{0}=6.3 \times 10^{-27} \mathrm{~g} \mathrm{~cm}^{-3}, r_{\mathrm{c}}=$ $0.26 \mathrm{Mpc}$, and $\beta=0.7$, we computed that at the present position of NGC $4848(0.75 \mathrm{Mpc}$ from the center $)$ the IGM density is $\rho_{0.75}=6 \times 10^{-28} \mathrm{~g} \mathrm{~cm}^{-3}$.

The radius at which ram pressure becomes efficient can be estimated as (Domainko et al. 2006)

$R_{\text {strip }}=0.5 R_{\mathrm{HI}} \ln \left(\frac{G M_{\mathrm{star}} M_{\mathrm{HI}}}{v^{2} \rho_{\mathrm{IGM}} 2 \pi R_{\mathrm{star}}^{2} R_{\mathrm{HI}}^{2}}\right)$, 
while the stripped mass is

$M_{\text {strip }}=M_{\mathrm{HI}}\left(\frac{R_{\text {strip }}}{R_{\mathrm{HI}}}+1\right) \exp \left(-\frac{R_{\text {strip }}}{R_{\mathrm{HI}}}\right)$.

In this calculation, we assumed exponential profiles for both the stars and the interstellar gas, with $R_{\text {star }}=5.0 \mathrm{kpc}$ (computed on the $H$ band image) and $R_{\mathrm{HI}}=1.8 \times R_{\mathrm{star}}=9.0 \mathrm{kpc}$ for the HI disk (Boselli \& Gavazzi 2006). This yielded $R_{\text {strip }}=8.5 \mathrm{kpc}$ and $M_{\text {strip }}=4.7 \times 10^{9} M_{\odot} \sim 0.75 M_{\mathrm{HI} \text { orig. }}$. This is in remarkably good agreement with the mass of stripped gas $\left(3.6 \times 10^{9} M_{\odot}\right)$ computed in Sect. 4, which in turns is consistent with the missing mass of atomic hydrogen $\left(4.1 \times 10^{9} M_{\odot}\right)$ computed from the HI deficiency parameter.

Using the ram pressure simulation by Kapferer et al. (2009) (the case with $V_{\text {rel }}=1000 \mathrm{~km} \mathrm{~s}^{-1}$ ) and assuming the IGM density computed above $\left(\rho_{0.75}=6 \times 10^{-28} \mathrm{~g} \mathrm{~cm}^{-3}\right)$, we derived that $65 \%$ of the original HI content is stripped in about $200 \mathrm{Myr}$. In spite of the different conditions found in the Virgo cluster with respect to the Coma cluster, a similarly short timescale $(\sim 100 \mathrm{Myr})$ is reported by Boselli et al. (2006) for an almost complete ablation of the atomic gas from NGC 4569. This time is significantly shorter than the crossing time in the Coma cluster $\left(1.6 \times 10^{9} \mathrm{yr}\right.$, Boselli \& Gavazzi 2006), supporting the conclusion that NGC 4848 is on its first passage through the cluster core. This time would be sufficient to remove most of its gas, in contradiction to the $1 \mathrm{Gyr}$ timescale proposed by Vollmer et al. (2001).

Acknowledgements. We acknowledge useful discussions with Massimo Dotti. We thank an anonymous referee for providing us with constructive comments and suggestions. This work made extensive use of GoldMine, the Galaxy On Line Database (http://goldmine.mib.infn.it). G. Gavazzi acknowledges financial support from Italian MIUR PRIN contract 200854ECE5. The present study could not have been conceived without the DR7 of SDSS. Funding for the Sloan Digital Sky Survey (SDSS) and SDSS-II has been provided by the Alfred P. Sloan Foundation, the Participating Institutions, the National Science Foundation, the US Department of Energy, the National Aeronautics and Space Administration, the Japanese Monbukagakusho, and the Max Planck Society, and the Higher Education Funding Council for England. The SDSS Web site is http://www.sdss.org/. The SDSS is managed by the Astrophysical Research Consortium (ARC) for the Participating Institutions. The Participating Institutions are the American Museum of Natural History, Astrophysical Institute Potsdam, University of Basel, University of Cambridge, Case Western Reserve University, The University of Chicago, Drexel University, Fermilab, the Institute for Advanced Study, the Japan Participation Group, The Johns Hopkins University, the Joint Institute for Nuclear Astrophysics, the Kavli Institute for Particle Astrophysics and Cosmology, the Korean Scientist Group, the Chinese Academy of Sciences (LAMOST), Los Alamos National Laboratory, the Max-Planck-Institute for Astronomy (MPIA), the Max-Planck-Institute for Astrophysics (MPA), New Mexico State University, Ohio State University, University of Pittsburgh, University of Portsmouth, Princeton University, the United States Naval Observatory, and the University of Washington.

\section{References}

Abazajian, K. N., Adelman-McCarthy, J. K., Agüeros, M. A., et al. 2009, ApJS, 182,543

Amram, P., Le Coarer, E., Marcelin, M., et al. 1992, A\&AS, 94, 175

Arrigoni Battaia, F., Gavazzi, G., Fumagalli, M., et al. 2012, A\&A, 543, A112

Becker, R. H., White, R. L., \& Helfand, D. J. 1994, Astronomical Data Analysis Software and Systems III, 1,165

Bekki, K., \& Couch, W. J. 2003, ApJ, 596, L13

Bell, E. F. 2003, ApJ, 586, 794

Bell, E. F., McIntosh, D. H., Katz, N., \& Weinberg, M. D. 2003, ApJS, 149, 289

Biviano, A., Katgert, P., Mazure, A., et al. 1997, A\&A, 321, 84

Boselli, A. 2011, A Panchromatic View of Galaxies, by Alessandro Boselli. (Berlin: Wiley-VCH)

Boselli, A., \& Gavazzi, G. 2006, PASP, 118, 517
Boselli, A., Lequeux, J., \& Gavazzi, G. 2002, A\&A, 384, 33

Boselli, A., Boissier, S., Cortese, L., et al. 2006, ApJ, 651, 811

Boselli, A., Boissier, S., Cortese, L., et al. 2009, ApJ, 706, 1527

Boselli, A., Hughes, T. M., Cortese, L., Gavazzi, G., \& Buat, V. 2012, A\&A, submitted

Bothun, G. D., \& Dressler, A. 1986, ApJ, 301, 57

Bravo-Alfaro, H., Cayatte, V., van Gorkom, J. H., \& Balkowski, C. 2000, AJ, 119,580

Bravo-Alfaro, H., Cayatte, V., van Gorkom, J. H., \& Balkowski, C. 2001, A\&A, 379,347

Cavaliere, A., \& Fusco-Femiano, R. 1978, A\&A, 70, 677

Cayatte, V., Kontanyi, C., Balkowski, C., \& Van Gorkom, J. H. 1994, AJ, 107, 1003

Chung, A., van Gorkom, J. H., Kenney, J. D. P., \& Vollmer, B. 2007, ApJ, 659, L115

Combes, F., Dupraz, C., Casoli, F., \& Pagani, L. 1988, A\&A, 203, L9

Condon, J. J., Cotton, W. D., \& Broderick, J. J. 2002, AJ, 124, 675

Conselice, C. J. 2003, ApJS, 147, 1

Cortese, L., Boselli, A., Franzetti, P., et al. 2008, MNRAS, 386, 1157

de Vaucouleurs, G., de Vaucouleurs, A., Corwin, H. G., Jr., et al. 1991 (Berlin Heidelberg New York: Springer-Verlag), 1

Domainko, W., Mair, M., Kapferer, W., et al. 2006, A\&A, 452, 795

Fumagalli, M., \& Gavazzi, G. 2008, A\&A, 490, 571

Fumagalli, M., Gavazzi, G., Scaramella, R., \& Franzetti, P. 2011, A\&A, 528, A46

Gavazzi, G. 1989, ApJ, 346, 59

Gavazzi, G., Boselli, A., Mayer, L., et al. 2001, ApJ, 563, L23

Gavazzi, G., Boselli, A., Donati, A., Franzetti, P., \& Scodeggio, M. 2003, A\&A, 400,451

Gavazzi, G., Zaccardo, A., Sanvito, G., Boselli, A., \& Bonfanti, C. 2004, A\&A, 417,499

Gavazzi, G., Savorgnan, G., \& Fumagalli, M. 2011, A\&A, 534, A31

Gavazzi G., Fumagalli, M., Galardo, V., et al. 2012, A\&A, in press, DOI: $10.1051 / 0004-6361 / 201218788$

Gunn, J. E., \& Gott, J. R. 1972, ApJ, 176, 1

Giovanelli, R., \& Haynes, M. 1985, ApJ, 292, 404

Helou, G., Soifer, B. T., \& Rowan-Robinson, M. 1985, ApJ, 298, L7

Iglesias-Páramo, J., Boselli, A., Cortese, L., Vílchez, J. M., \& Gavazzi, G. 2002, A\&A, 384, 383

Iglesias-Páramo, J., Boselli, A., Gavazzi, G., \& Zaccardo, A. 2004, A\&A, 421, 887

Kapferer, W., Sluka, C., Schindler, S., Ferrari, C., \& Ziegler, B. 2009, A\&A, 499, 87

Kenney, J. D. P., \& Young, J. S. 1989, ApJ, 344, 171

Kenney, J. D. P., Tal, T., Crowl, H. H., Feldmeier, J., \& Jacoby, G. H. 2008, ApJ, 687, L69

Kennicutt, R. C., Jr. 1998, ApJ, 498, 541

Martin, D. C., Fanson, J., Schiminovich, D., et al. 2005, ApJ, 619, L1

Mohr, J. J., Mathiesen, B., \& Evrard, A. E. 1999, ApJ, 517, 627

Neugebauer, G., Habing, H. J., van Duinen, R., et al. 1984, ApJ, 278, L1

Osterbrock, D. 1989, Astrophysics of gaseous nebulae and active galactic nuclei, University Science Books

Ruszkowski, M., Bruggen, M., Lee, D., \& Shin, M.-S. 2012, ApJ, submitted [arXiv: 1203.1343]

Schlegel, D. J., Finkbeiner, D. P., \& Davis, M. 1998, ApJ, 500, 525

Smith, R. J., Lucey, J. R., Hammer, D., et al. 2010, MNRAS, 408, 1417

Spector, O., Finkelman, I., \& Brosch, N. 2012, MNRAS, 419, 2156

Spitzer, L. 1978 (New York: Wiley-Interscience), 333

Sun, M., Donahue, M., \& Voit, G. M. 2007, ApJ, 671, 190

Tonnesen, S., \& Bryan, G. L. 2009, ApJ, 694, 789

Tonnesen, S., \& Bryan, G. L. 2010, ApJ, 709, 1203

Tonnesen, S., \& Bryan, G. L. 2012, MNRAS, 422, 1609

Vollmer, B., Braine, J., Balkowski, C., Cayatte, V., \& Duschl, W. J. 2001, A\&A, 374,824

Wright, E. L., Eisenhardt, P. R. M., Mainzer, A. K., et al. 2010, AJ, 140, 1868

Yagi, M., Komiyama, Y., Yoshida, M., et al. 2007, ApJ, 660, 1209

Yagi, M., Yoshida, M., Komiyama, Y., et al. 2010, AJ, 140, 1814

Yoshida, M., Yagi, M., Okamura, S., et al. 2002, ApJ, 567, 118

Yoshida, M., Ohyama, Y., Iye, M., et al. 2004, AJ, 127, 90

Yoshida, M., Yagi, M., Komiyama, Y., et al. 2008, ApJ, 688, 918

Yoshida, M., Yagi, M., Komiyama, Y., et al. 2012, ApJ, 749, 43

Zabludoff, A. I., Geller, M. J., Huchra, J. P., \& Vogeley, M. S. 1993, AJ, 106, 1273

Zwicky, F., Herzog, E., Karpowicz, M., Kowal, C., \& Wild, P. 1961-1968, Catalogue of Galaxies and Clusters of Galaxies, Pasadena, CalTech (CGCG) 